\title{
mRNA and miRNA Expression Analysis Reveal the Regulation for Flower Spot Patterning in Phalaenopsis 'Panda'
}

\author{
Anjin Zhao ${ }^{1}$, Zheng Cui ${ }^{1}$, Tingge Li ${ }^{1}$, Huiqin Pei ${ }^{1}$, Yuhui Sheng ${ }^{1}$, Xueqing Li ${ }^{1}$, Ying Zhao ${ }^{1,2}$, \\ Yang Zhou ${ }^{1,2}$, Wenjun Huang ${ }^{3}$, Xiqiang Song ${ }^{1,2}$, Ting Peng ${ }^{4}$ and Jian Wang ${ }^{1,2, *}$ \\ 1 Key Laboratory of Ministry of Education for Genetics and Germplasm Innovation of Tropical Special Trees \\ and Ornamental Plants, Hainan Key Laboratory for Biology of Tropical Ornamental Plants Germplasm, \\ College of Forestry, Hainan University, Haikou 570228, China \\ 2 Research Center for Terrestrial Biodiversity of the South China Sea, College of Forestry, Hainan University, \\ Haikou 570228, China \\ 3 Department of Development and Design, Hainan University, Haikou 570228, China \\ 4 Key Laboratory of Germplasm Innovation on Protection and Conservation of Mountain Plant Resources, \\ Ministry of Education/College of Agriculture, Guizhou University, Guiyang 550025, China \\ * Correspondence: wjhainu@hainanu.edu.cn; Tel.: +86-6627-7907
}

Received: 14 July 2019; Accepted: 26 August 2019; Published: 30 August 2019

\begin{abstract}
Phalaenopsis cultivar 'Panda' is a beautiful and valuable ornamental for its big flower and unique big spots on the petals and sepals. Although anthocyanins are known as the main pigments responsible for flower colors in Phalaenopsis, and the anthocyanins biosynthetic pathway in Phalaenopsis is generally well known, the detailed knowledge of anthocynins regulation within the spot and non-spot parts in 'Panda' flower is limited. In this study, transcriptome and small RNA libraries analysis from spot and non-spot sepal tissues of 'Panda' were performed, and we found PeMYB7, PeMYB11, and miR156g, miR858 is associated with the purple spot patterning in its sepals. Transcriptome analyses showed a total 674 differentially expressed genes (DEGs), with 424 downregulated and 250 upregulated (Non-spot-VS-Spot), and 10 candidate DEGs involved in anthocyanin biosynthetic pathway. The qPCR analysis confirmed that seven candidate structure genes (PeANS, PeF3'H, PeC4H, PeF3H, PeF3H1, Pe4CL2, and PeCHI) have significantly higher expressing levels in spot tissues than non-spot tissues. A total 1552 differentially expressed miRNAs (DEMs) were detected with 676 downregulated and 876 upregulated. However, microRNA data showed no DEMs targeting on anthocyanin biosynthesis structure gene, while a total 40 DEMs target transcription factor (TF) genes, which expressed significantly different level in spot via non-spot sepal, including 2 key MYB regulator genes. These results indicated that the lack of anthocyanidins in non-spot sepal may not directly be caused by microRNA suppressing anthocyanidin synthesis genes rather than the MYB genes. Our findings will help in understanding the role of miRNA molecular mechanisms in the spot formation pattern of Phalaenopsis, and would be useful to provide a reference to similar research in other species.
\end{abstract}

Keywords: Phalaenopsis; transcriptome; microRNA; anthocyanin biosynthesis; molecular mechanism

\section{Introduction}

Flower spots are heterochromatic dots or streaks with a specific texture and pattern appearing on the corollas of plants, which can affect the behavior of pollinators and the ornamental value of flowers [1-4]. Previous studies have confirmed the flower spot is caused by the accumulation of anthocyanins in a specific area of corollas. For example, peonidin-3-O-glucoside, malvidin-3-O-glucoside, 
delphinidin-3-O-diglucoside, and cyanidin-3-O-glucoside are the main anthocyanins found in petal and sepal spots in Oncidium [5], and cyanidin and delphinidin are the main anthocyanidins in the spot of pansy (Viola $\times$ wittrockiana Gams.) petals [6].

Molecular mechanism of anthocyanins accumulation has been clearly studied in some plants, such as Dianthus hybrida [7], Antirrhinum majus [8], Petunia hibrida [9], Platycodonis Radix [10], Gerbera jamesonii [11], and Phalaenopsis equestris [12], and the anthocyanin pathway, which is a branch of the flavonoid pathway, has been elucidated as well $[13,14]$. Three kinds of transcription factor genes families-MYB transcription factor, bHLH transcription factor, and WD40 repeat protein family (MBW)—-were also found to regulate the anthocyanin biosynthesis genes [15].

As for the accumulation of anthocyanin in specific regions of colloras, the direct cause attributes to the specific expression of the biosynthesis genes involved in the anthocyanin pathway. For example, high level expression of the genes of $\mathrm{OgCHI}$ and OgDFR results in anthocyanin accumulation and pigmented spot formation in yellow lip in Oncidium [5,16]. Upregulation of LhCHSA, LhCHSB, and $L h D F R$ is detected within the spots located in the center of the petals, in comparison to the low expression levels in the margin in Lilium 'Sorbonne' [17]. In Dendrobium moniliforme, pigment accumulation in the base of the column has been caused by a consequence of preferential expression of $D m F 3^{\prime} 5^{\prime} H$ [18]. In Clarkia gracilis, precise spatiotemporal regulation of the expression of the anthocyanin genes $F 3^{\prime} H, F 3^{\prime} 5^{\prime} H, D F R 1$, and DFR2 produces spotted petals [19]. In pansy, VwDFR, $V w F 3^{\prime} 5^{\prime} H$ and $V w A N S$ have more significantly higher level expression in cyanic flower areas [6]. Moreover, the MYB genes also play an important role for the production of flower spot by regulating the anthocyanin biosynthesis genes. For example, the large purple spots in Phalaenopsis 'Everspring Fairy' was mainly caused by the expression of MYB [12], and the LhMYB6 and LhMYB12 positively regulate anthocyanin biosynthesis and determine organ- and tissue-specific accumulation of anthocyanin in Asiatic hybrid lily 'Montreux' [20]. Hsu also conducted a detailed study on three MYBs in Phalaenopsis, and found that the color patterning of flower sepals, petals and lips is regulated by different MYB genes combinations, and the pigmented veins and spots on the petals are also regulated by these three MYB genes [21].

RNA interference, regulating gene expression by post-transcriptional mechanisms, has also been recognized to play an important role in the color special patterning model of some plants [22]. Koseki et al. [23] found that the star-type color pattern of Petunia hybrida Red star' flowers is induced by sequence-specific degradation of chalcone synthase RNA. Their further study found that the formation of bicolor flower types of petunia was due to RNA interference in two CHSA alleles (PhCHS-A1 and PhCHS-A2) [23]. In Arabidopsis thaliana, miRNAs may function as regulators in anthocyanin biosynthesis by targeting on related transcription factors and lead to the different accumulation of anthocyanin [24].

Phalaenopsis spp. have become important ornamental plants worldwide for their long-lasting and various colorful flowers [25]. There are varieties of flower colors and corolla pigmentation patterning styles of Phalaenopsis spp., so it is very important to find more details about the flower color patterning for its breeding and production. However, there was little research in this field because of the complex anthocyanin synthesis pathways and the gene expression networks in Phalaenopsis spp. RNA sequencing can effectively identify the subtle differences in gene expression and find the targeted genes of small RNA in transcription level, so it has been used to study flower color patterning recently, such as in Lilium 'Tiny Padhye' [26], monkeyflowers (Mimulus) [27], and tree peony [28]. In this study, the transcriptome sequencing and small RNA sequencing in spot and non-spot sepal of Phalaenopsis 'Panda' were carried out by genomics Illumina sequencing platform, and the expression levels of candidate genes, as well as microRNAs, were verified by qPCR. Meanwhile, the model of flower spot formation pattern was predicted by the interactions of the structural genes, regulatory genes together with small RNA. This study created a joint research of transcriptome sequencing and small RNA sequencing to explain the spot pigmentation in Phalaenopsis. spp., and the results will be helpful for the breeding of new colorful cultivars of Phalaenopsis spp. 


\section{Results}

\subsection{Anthocyanin Accumulation Patterns in Phalaenopsis 'Panda'}

The examination by light microscope showed that anthocyanin accumulated in the upper epidermal cells of spot area, while no anthocyanin accumulated in cells in non-spot area (Figure 1A-C). This result indicated that the visible spots resulted from accumulation of anthocyanin in cells of the upper epidermis. Furthermore, the anthocyanin content of the spot in Phalaenopsis 'panda' sepals was significantly different from that of the sepal according to the results by the UV-visible spectrophotometer scanning detection (Figure 1D,E). Anthocyanin content measured by the $\mathrm{pH}$ differential method revealed that high content of anthocyanin $(1.0798 \mathrm{mg} / \mathrm{g})$ was accumulated in spot area, while was barely detectable in the acyanic parts extraction.
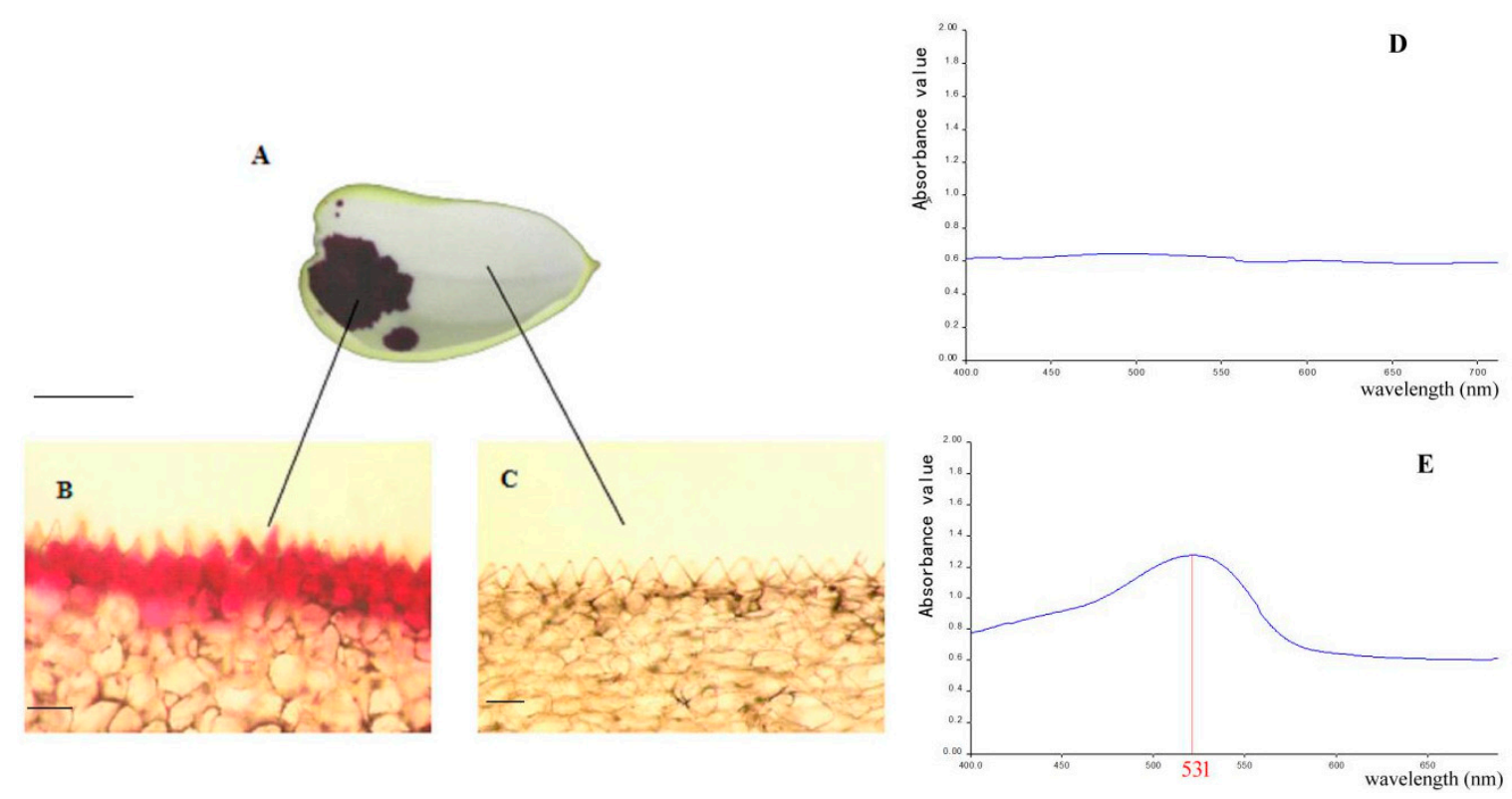

Figure 1. Anatomical structures and anthocyanin content of Phalaenopsis 'Panda' sepal. (A) Sepal (bar = $1 \mathrm{~cm}$ ); (B) Upper epidermal cell in spot area (bar = $10 \mu \mathrm{m}) ;(\mathbf{C})$ Upper epidermal cell in non-spot area (bar = $10 \mu \mathrm{m})$; (D) UV-visible spectrophotometer scanning of non-spot area; (E) UV-visible spectrophotometer scanning of spot area. Red number of $531 \mathrm{~nm}$ represents the absorption wavelength of anthocyanin.

\subsection{Construction of cDNA Library and Gene Mapping to the Reference Genomes}

Four cDNA libraries were constructed using Illumina Hiseq 2000 platform (Illumina, San Diego, CA, USA) and 44,632,028, 45,110,536, 45,380,086, 44,434,116 high-quality reads were obtained, respectively (Table S1 in supplememntary materials). The sequencing raw data has been deposited into the Short Reads Archive (SRA) database under the accession number SRP166213. These clean reads were mapped to reference genome of Phalaenopsis equestris and the average gene mapping ratio of each sample was $55.47 \%$. We considered that the low homology between Phalaenopsis 'Panda' and Phalaenopsis equestris result to the low mapping ratio, but it has no effect on RNAseq quantitative analysis owing to the high clean reads quantity and enough sequencing data (Table S2).

\subsection{Functional Annotation and Classification}

To annotate the gene with putative functions, the assembled genes were searched against the public databases of NR. Among them, 17,871 genes were annotated to the NR database. To further illustrate the main biological functions of the transcripts, GO (15,588 genes) and KEGG pathway $(19,864$ genes) analyses were performed (Table S3). 


\subsection{Identification of Differentially Expressed Genes and KEGG Enrichment Analysis of DEGs}

A total of 674 DEGs were detected in pairwise comparison with 424 downregulated and 250 upregulated genes (Non-spot-VS-Spot) (Figure 2). A total of 543 DEGs were mapped to all 106 pathways. Notably, the Flavonoid biosynthesis (ko00941, 12 DEGs of 105 gene with $Q$-value $=0.0005279107$ ) and Phenylalanine metabolism (ko00360, 8 DEGs of 132 gene with $Q$-value $=1.900591 \times 10^{-1}$ ) were most significantly enriched in top20 pathways (Figure 3, Table S4).

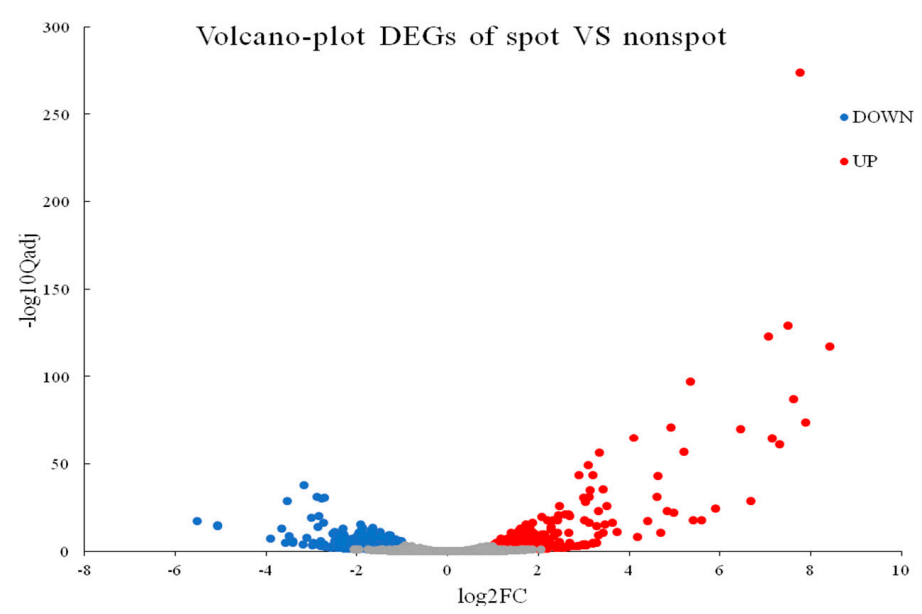

Figure 2. Volcano plot of differentially expressed genes (DEGs). X axis: $\log 2$ transformed fold change; Y axis: - $\log 10$ transformed significance; Red points: upregulated DEGs; Blue points: downregulated DEGs. Gray points: non-DEGs.

The most enrichment pathway

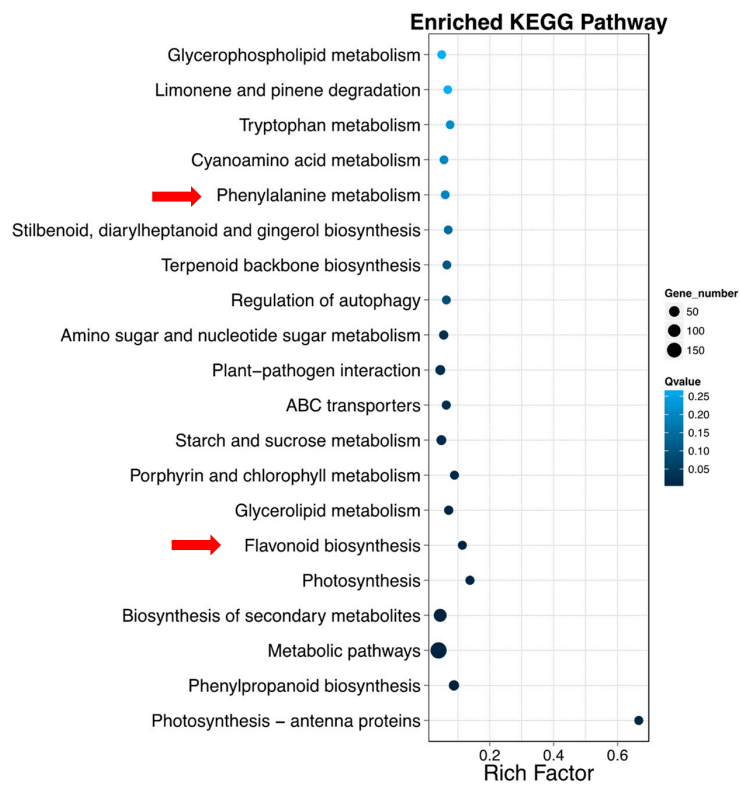

Figure 3. The most enrichment pathway of DEGs (TOP20). $X$ axis: enrichment factor; $Y$ axis: pathway name; The color: the q-value (high: white, low: blue), the lower q-value indicates the more significant enrichment; Point size: DEG number (The bigger dots refer to larger amount); Rich Factor: the value of enrichment factor, which is the quotient of foreground value (the number of DEGs) and background value (total Gene amount), the larger the value, the more significant enrichment. Red arrows represent the pathways related directly to the anthocyanin biosynthesis. 


\subsection{DEGs in Anthocyanin Biosynthesis and MBW Genes}

There were 10 DEGs in flavonoid biosynthesis which are directly related to spot pattern, including PeANS (PEQU_25924), PeF3'H (PEQU_00400), PeC4H (PEQU_12025), PeDFR (PEQU_34933), PeCHI (PEQU_22606), PeF3H1 (PEQU_38891), PeF3H (PEQU_22432), PePAL (PEQU_01877), Pe4CL2 (PEQU_00756), Pe4CL (PEQU_07458), and most of them presented higher expression level in spot areas than non-spot areas (Table 1, Figure 4).

Table 1. Putative anthocyanin structural genes identified from differentially expressed genes (DEGs).

\begin{tabular}{ccccccc}
\hline Gene ID & Annotation & $\begin{array}{c}\text { FPKM } \\
\text { (non-spot) }\end{array}$ & $\begin{array}{c}\text { FPKM } \\
\text { (spot) }\end{array}$ & Log2FC & Padj & Up/Downregulation \\
\hline PEQU_25924 & PeANS & 119.9303758 & $21,612.25078$ & 7.493508409 & $4.71 \times 10^{-130}$ & $\mathrm{Up}$ \\
PEQU_00400 & PeF3'H & 31.02321 & 6117.42 & 7.623432 & $5.88 \times 10^{-88}$ & $\mathrm{Up}$ \\
PEQU_12025 & PeC4H & 2272.365 & 8325.431 & 1.87333 & $1.79 \times 10^{-8}$ & $\mathrm{Up}$ \\
PEQU_34933 & PeDFR & 17.40537 & 5973.723 & 8.422954 & $1.13 \times 10^{-117}$ & $\mathrm{Up}$ \\
PEQU_22606 & PeCHI & 2467.193 & 707.8831 & -1.80129 & $1.13 \times 10^{-6}$ & Down \\
PEQU_07458 & Pe4CL & 11.87304 & 253.4331 & 4.415844 & $3.15 \times 10^{-18}$ & $\mathrm{Up}$ \\
PEQU_38891 & PeF3H1 & 52.59874 & 5401.439 & 6.682172 & $2.59 \times 10^{-29}$ & $\mathrm{Up}$ \\
PEQU_22432 & PeF3H & 21.49244 & 3076.48 & 7.161307 & $2.17 \times 10^{-65}$ & $\mathrm{Up}$ \\
PEQU_01877 & PePAL & 14783.45 & 6368.878 & -1.21487 & $8.83 \times 10^{-8}$ & Down \\
PEQU_00756 & Pe4CL2 & 383.56 & 1290.653 & 1.750578 & 0.000153 & $\mathrm{Up}$ \\
\hline
\end{tabular}
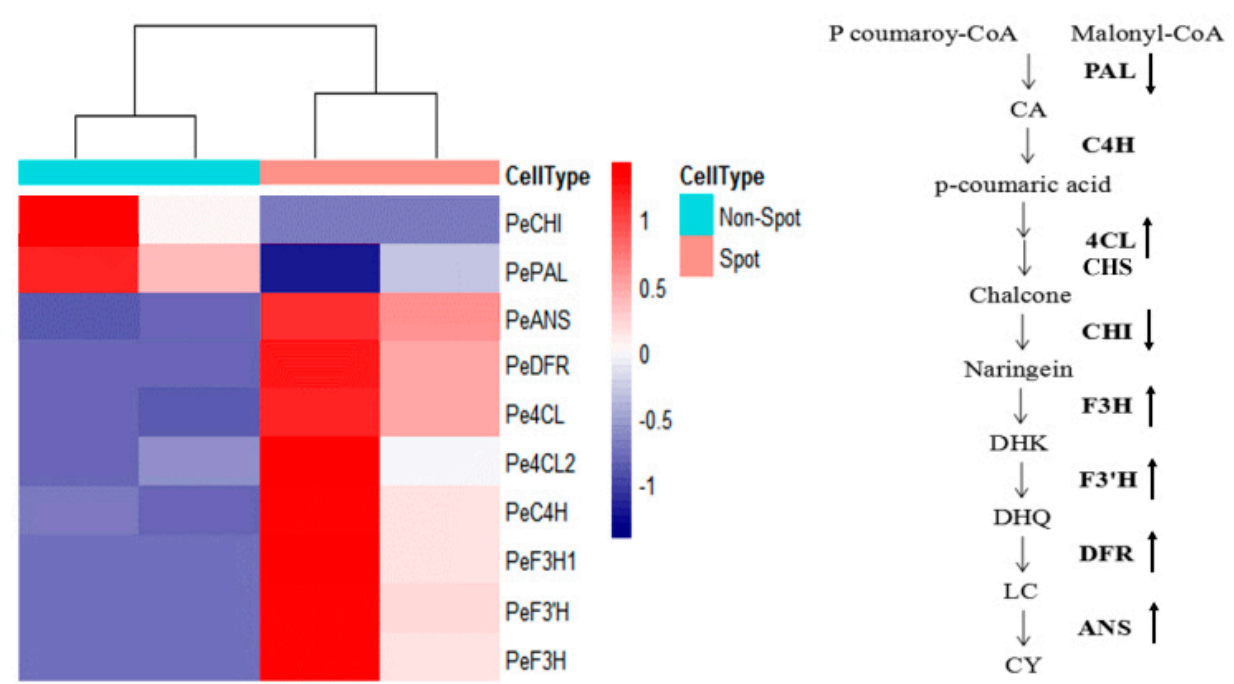

Figure 4. Heatmap of differentially expressed structure genes (DEGs) related to anthocyanin biosynthesis in Phalaenopsis. Bold arrow means up/downregulations of genes. (PAL, phenylalanine ammonia lyase; $\mathrm{C} 4 \mathrm{H}$, cinnamate-4-hydroxylase; $4 \mathrm{CL}$, 4-coumarate-CoA ligase; $\mathrm{CHS}$, chalcone synthase; $\mathrm{CHI}$, chalcone isomerase; F3H, flavanone 3-hydroxylase; F3' $\mathrm{H}$, Flavonoid 3'-hydroxylase; DFR, dihydroflavonol 4-reductase; ANS, Anthocyanidin synthase; DHK, dihydrokaempferol; DHQ, dihydroquercetin; DHM, dihydromyricetin; LC, leucocyanidin; $\mathrm{Cy}$, cyaniding).

As for the MBW transcriptor genes related to the anthocyanin synthesis, 4 unigenes were found having significant expression difference including 3 MYB uingenes and $1 \mathrm{bHLH}$ unigene (Table 2). In these transcriptor unigenes, 2 MYB unigenes and $1 \mathrm{bHLH}$ unigene were upregulated and $1 \mathrm{MYB}$ unigene was downregulated. 
Table 2. Putative MBW genes identified from differentially expressed genes (DEGs).

\begin{tabular}{ccccccc}
\hline Gene ID & Annotation & $\begin{array}{c}\text { FPKM } \\
\text { (non-spot) }\end{array}$ & $\begin{array}{c}\text { FPKM } \\
\text { (spot) }\end{array}$ & Log2FC & Padj & Up/Downregulation \\
\hline PEQU_03393 & PeMYB7 & 29.41538126 & 113.6604505 & 0.04965 & 0.00303 & UP \\
PEQU_10361 & PeMYB11 & 10.58835723 & 1693.628622 & 7.32149 & $8.71 \times 10^{-62}$ & UP \\
PEQU_10362 & PeMYB11 & 4.49564678 & 216.34208328 & 5.58864183 & $2.35 \times 10^{-18}$ & UP \\
PEQU_09064 & PeMYB16 & 1022.11354 & 508.0418102 & -1.00853 & 0.03605 & DOWN \\
PEQU_19747 & PebHLH1 & 149.2074009 & 801.9464953 & 2.42618 & $2.57 \times 10^{-18}$ & UP \\
\hline
\end{tabular}

\subsection{Data Analysis of Small RNA Sequencing}

Four sRNA libraries were constructed and 23,352,936, 24,896,769, 26,605,438, 25,926,370 high-quality tag were obtained, respectively (Table S5). The sequencing raw data has been deposited into the Short Reads Archive (SRA) database under the accession number SRP161646. These clean tags were mapped to reference genome of Phalaenopsis equestris and other sRNA databases. The average gene mapping ratio of each sample was $77.38 \%$ (Table S6).

A total of 1552 DEMs were detected in pairwise comparison with 676 downregulated and 876 upregulated (Non-spot-VS-Spot) (Figure 5). miRNA target gene prediction resulted in 1396 common unigenes between TargetFinder (http://http://targetfinder.org/) and psRobot (http://http://omicslab.genetics.ac.cn/psRobot/), 1648 by TargetFinder and 7652 by psRobot, and totally 1307 target unigenes were aligned to KEGG database. However, there were no DEMs were found targeting on anthocyanin biosynthesis structure gene. As for the regulator genes, we found totally 12 microRNAs targeting on 10 significant different expressed MYB, bHLH or WRKY unigenes (Table 3). However, only PeMYB7 (PEQU_03393), PeMYB11 (PEQU_10361, PEQU_10362) were significantly upregulated in these TF families according to the transcriptomes data, and these microRNAs belonged to miR156 or miR858 families.

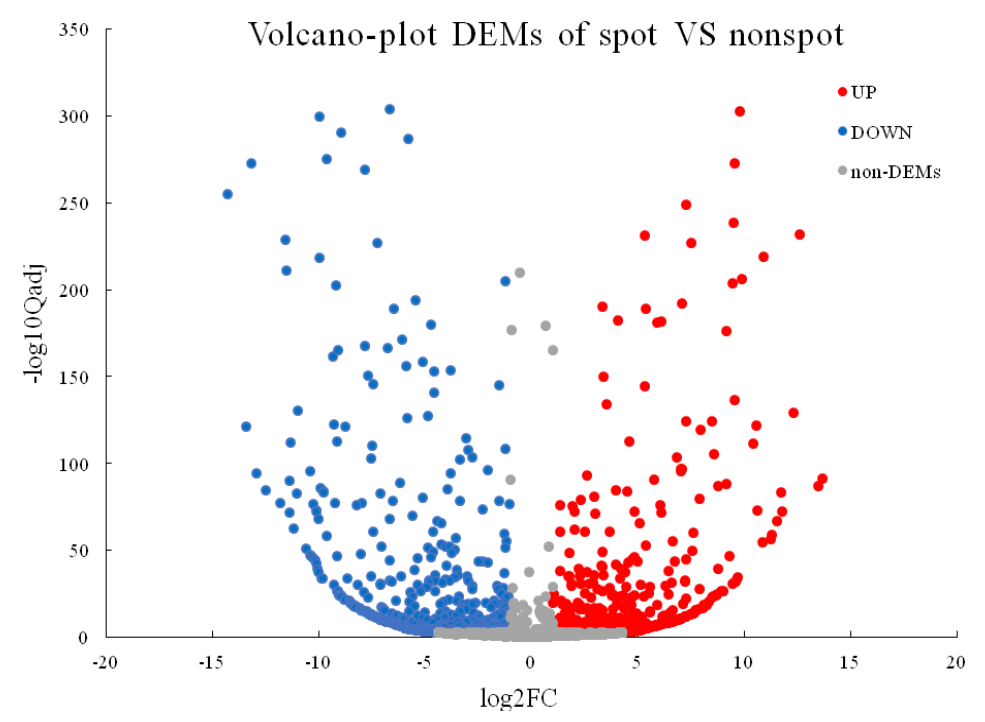

Figure 5. Volcano plot of DEMs. X axis represents $\log 2$ transformed fold change. $\mathrm{Y}$ axis represents - $\log 10$ transformed significance. Red points represent upregulated DEMs. Blue points represent downregulated DEMs. Gray points represent non-DEMs. 
Table 3. Putative miRNAs targeting on $M Y B, b H L H$ or WRKY unigenes.

\begin{tabular}{|c|c|c|c|c|c|c|c|}
\hline miRNA id & $\begin{array}{c}\text { miRNA } \\
\text { Expression in } \\
\text { Non-spot }\end{array}$ & $\begin{array}{l}\text { miRNA } \\
\text { Expression } \\
\text { in Spot }\end{array}$ & $\begin{array}{l}\text { Up/Down } \\
\text { Regulation } \\
\text { of miRNA }\end{array}$ & $p$-Value & Target Gene & $\begin{array}{c}\text { Target } \\
\text { Unigene ID }\end{array}$ & $\begin{array}{l}\text { Up/Downregulation } \\
\text { of Target Unigene } \\
\text { in Spot }\end{array}$ \\
\hline Novel-m1700-5p & 12 & 0 & UP & 0.000818887 & РeMYB39 & PEQU_22029 & NA \\
\hline Novel-m0210-3p & 82 & 10 & UP & $1.69 \times 10^{-13}$ & PeMYB7 & PEQU_03393 & UP \\
\hline \multirow{2}{*}{ cme-miR858 } & \multirow{2}{*}{52} & \multirow{2}{*}{0} & \multirow{2}{*}{ UP } & \multirow{2}{*}{$1.57 \times 10^{-14}$} & PeMYB11 & PEQU_10361 & UP \\
\hline & & & & & PeMYB11 & PEQU_10362 & UP \\
\hline \multirow{3}{*}{ ath-miR858 } & \multirow{3}{*}{336} & \multirow{3}{*}{116} & \multirow{3}{*}{ UP } & \multirow{3}{*}{$4.67 \times 10^{-34}$} & PeMYB11 & PEQU_10361 & UP \\
\hline & & & & & PeMYB11 & PEQU_10362 & UP \\
\hline & & & & & PeMYB12 & PEQU_20333 & NA \\
\hline ata-miR528-3p & 12 & 0 & UP & 0.000818887 & PebHLH086 & PEQU_08299 & NA \\
\hline zma-miR528a-3p & 49 & 1 & UP & $2.66 \times 10^{-12}$ & PebHLH086 & PEQU_08299 & NA \\
\hline
\end{tabular}

\section{7. qPCR of Key Structural Genes, Regulate Genes and miRNA}

The expression levels of 10 anthocyanin genes and 4 transcriptor genes between the non-spot and spot areas were verified by qPCR. The results showed the genes Pe4CL2, PeANS, PeF3H, PeF3H1, $P e F 3^{\prime} H$ and PeMYB7, PeMYB11 presented significantly higher expression levels in spot areas than the non-spot areas (Figure 6), which was generally consistent with the results of the transcriptome data (Table 1).

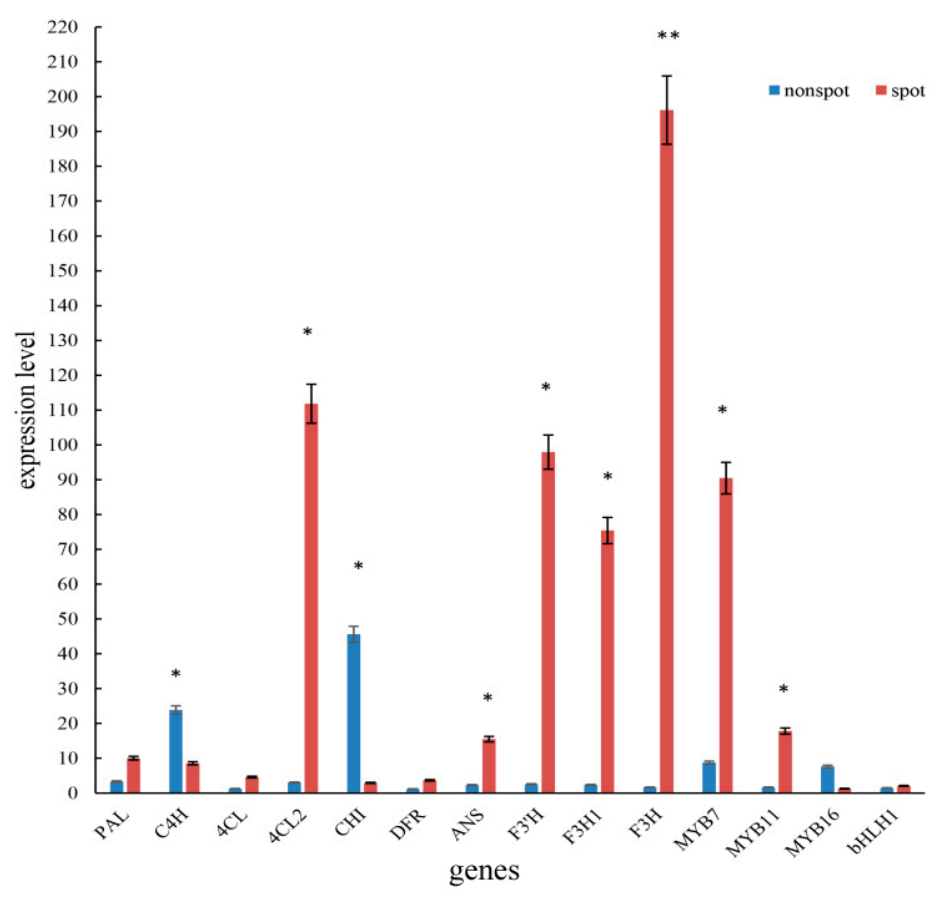

Figure 6. The expression level of structure gene and regulate gene Associated with Anthocyanin Biosynthesis, ${ }^{*}$ represent there are significant differences between spot and non-spot area; ${ }^{* *}$ represent there are extremely significant differences; red: spot area, blue: non-spot area.

The expression of microRNA of RNA miR858, miR156g were also analyzed by qPCR between the non-spot and spot areas (Figure 7). The results showed that they presented higher expression levels in non-spot areas, which means their targeting genes, PeMYB7 and PeMYB11, having less expression levels in the non-spot areas. 


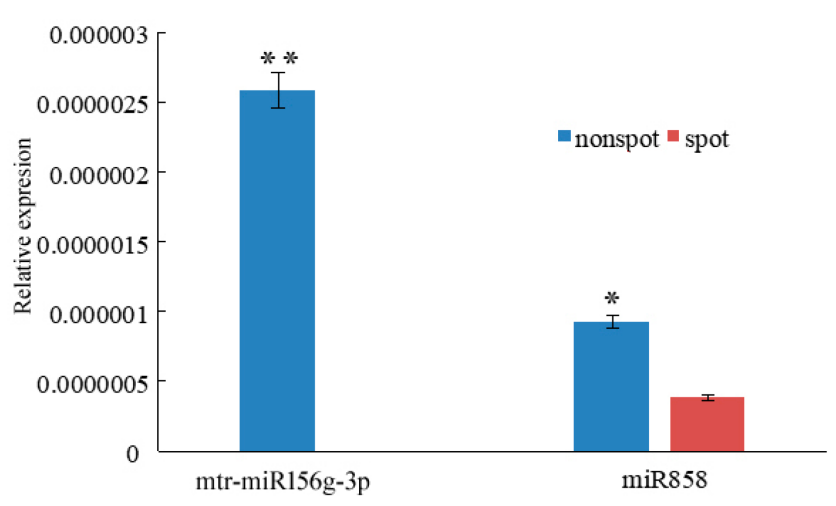

Figure 7. The expression level of mtr-miR156g-3p and miR858. ${ }^{* *}$ represent an extremly significant difference $(p<0.01) ;{ }^{*}$ represent an significant difference $(p<0.05)$.

\section{Discussion}

\subsection{Low Expression of Anthocyanin Genes Causing the Lack of Pigments in Non-Spot Areas}

In this study, we found anthocyanin accumulated in the upper epidermal cells in spot area, while no anthocyanin accumulated in cells in non-spot areas of 'Panda' (Figure 1). Transcriptome data and qPCR indicated 7 anthocyanin genes were low expression in the non-spot areas, especially for the very different expression levels of $4 C L 2, F 3^{\prime} H, F 3 H$ and F3H1 (Table 2, Figure 6). In Phalaenopsis 'Everspring Fairy', which also has purple spot on the white petal and sepal, DFR is the main gene which differently expressed between the purple and white part of the petals and sepals [12]. However, in Phalaenopsis 'Panda' expression of DFR in spot tissue was not significantly changed in comparison to non-spot area. These results showed that the same phenotype may be caused by different genes in the pigmentation in Phalaenopsis.

\subsection{PeMYB7 and PeMYB11 Are Important Genes in Spot Formation}

MYBs have been found to be very important in the floral pigmentation patterning in Phalaenopsis spp. In the sepals/petals, silencing of PeMYB2, PeMYB11, and PeMYB12 resulted in the loss of the full-red pigmentation, red spots, and venation patterns, respectively; PeMYB11 was responsive to the red spots in the callus of the lip, and PeMYB12 participated in the full pigmentation in the central lobe of the lip [21]. In P. amabilis and P. schilleriana, anthocyanin-specific Myc and Wd were expressed, however, Myb specific for anthocyanin biosynthesis were undetectable in P. amabilis; in Phalaenopsis 'Everspring Fairy' petals and sepals, high levels of anthocyanin-specific Myb transcripts were present in the purple, but not in the white sectors [12]. Hsu, et al. [29] verified PeMYB11 as the major regulatory R2R3-MYB transcription factor for regulating the production of the black color, and a retrotransposon, named Harlequin Orchid RetroTransposon 1 (HORT1), resulted in strong expression of PeMYB11 and thus extremely high accumulation of anthocyanins in the harlequin flowers of the $P$. Yushan Little Pearl variety.

In our study, PeMYB7 and PeMYB11 expressed significantly different between the spot and non-spot areas, while PeMYB2 and PeMYB12 had not different expression levels (Table 2 and Figure 6). This result indicated that the gene of PeMYB11 may also play an important role in spot pigmentation in Phalaenopsis 'Panda', which was similar to these previous researches [12,21,29,30]. PeMYB7 had different expression levels between the spot and non-spot areas of sepals in this research, which indicated this gene may also associate with purple spot patterning in Phalaenopsis 'Panda'. The function of PeMYB7 is not very clear presently, however, in the phylogenetic tree inferred from MYB genes of Phalaenopsis equestris and Oryza sativa (Figure 8A), PeMYB7 was in the same clad of PeMYB2 and PeMYB8, suggesting PeMYB7 may have similar function as PeMYB2 which can activate anthocyanin synthesis [30]. 


\section{3. miR156g, miR858 Silence PeMYB7, and PeMYB11}

MiRNAs can influence tissue anthocyanin formation in previous studies. In petunia, the sequence-specific degradation of CHS RNA is the primary cause of the formation of white sectors in 'Red Star' flowers [23]. In potato (Solanum tuberosum L.), small RNAs of miR828 and TAS4 D4(-) can decrease the expression levels of MYB12 and R2R3-MYB genes in purple skin and flesh, which caused to anthocyanin accumulation [31]. In our research, miR156 and miR858 were predicted as the main interference RNAs for PeMYB7 and PeMYB11 (Table 3). In order to confirm PeMYB7 and PeMYB11 are the direct targets of the small RNAs, we download the complete cDNAs of PeMYB7 (GenBank: KF769472.1) and PeMYB11 (GenBank: MH675649.1), and analyzed the target sites of miR156 and miR858 in these two genes (Figure 8B). This finding confirms that miR156g and miR858 can silence PeMYB7 and PeMYB11.

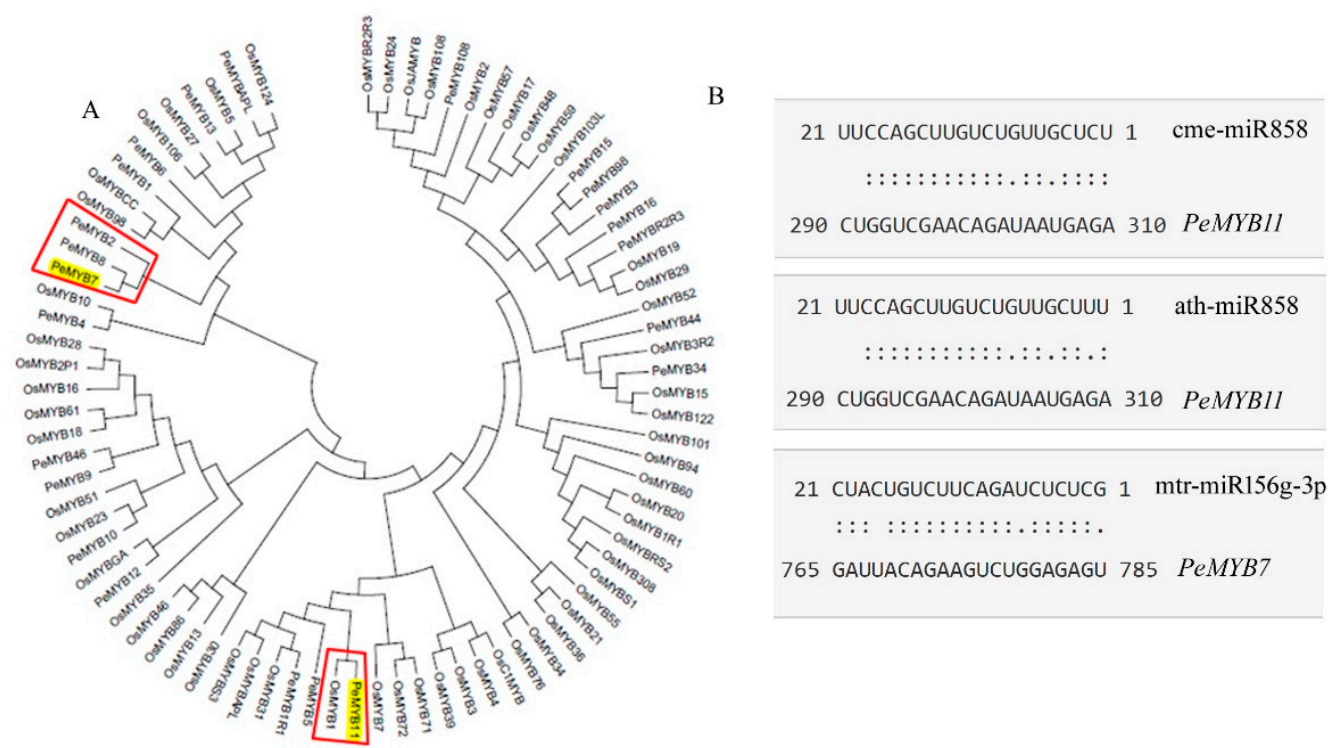

Figure 8. Identification of PeMYB7, PeMYB11 and their target sites by miR156g, miR858.

(A) Phylogenetic tree inferred from the MYB sequences of Phalaenopsis equestris and Oryza sativa;

(B) Identification of target sites of miR156 and miR858 in PeMYB7 and PeMYB11.

In Arabidopsis thaliana, miR156 negatively regulates the anthocyanin accumulation by regulating the expression level of target gene MYB113 indirectly [32]. High miR156 level promotes anthocyanin biosynthesis through the negative regulation of SPL9 gene because SPL9 can destabilize the MYB-bHLH-WD40 transcriptional activation complex. However, in this study, SPL9 gene in Phalaenopsis doesn't express differently, which indicated that mtr-miR156g-3p may be able to directly silence PeMYB7 and caused the anthocyanin lacking on the white sector.

The biological functions of miR858 has not been fully explored, however, small RNA sequencing in Arabidopsis thaliana [24] and Vitis vinifera L. [33] verified that its target gene is MYB12, which is a positive transcript factor for the anthocyanin synthesis. Chitwood et al. transferred the constructed binary vector into wild-type tomato plants to reduced MIR858 expression level, and found the expression levels of $S L Y-M y b 12$ in transgenic plants were lower than wild type, with upregulations of the related structure gene $P A L, D F R, A N S$ and CHS involved in anthocyanin biosynthesis, and increasing of anthocyanin content [34]. Ballester et al. [35] used VIGS technology silenced MYB12 and then found the tomato fruits turned pink. In our study the targeted genes of miR858 were predicted to be both MYB12 and MYB11 (Table 3), however, the unigene of PeMYB12 didn't show significantly different expression levels between the spot and non-spot areas, suggesting that miR858 explored higher effect on the unigene of PeMYB11 in 'Panda'. 


\subsection{A Proposed Modelsummarizing of Spot Formation Pattern in Phalaenopsis 'Panda'}

Our research confirmed $\mathrm{Pe} 4 \mathrm{CL} 2, \mathrm{PeF} 3 \mathrm{H}, \mathrm{PeF} 3^{\prime} \mathrm{H}$, and PeANS expression were low or repressed in non-spot areas in 'Panda' sepals, and MIR156g and MIR858 may silence the expression of PeMYB11 and PeMYB7 in non-spots parts. In conclusion, we illustrated a proposed molecular mechanism in spots formation in 'Panda' (Figure 9). The diagram revealed the unique spot formation in 'panda' may result from the higher expression of MIR156g and MIR858 clusters in non-spot tissue, which targeted and suppressed the expression of key regulate genes PeMYB7, PeMYB11 and then caused very low expression of $\mathrm{Pe} 4 \mathrm{CL} 2, \mathrm{PeF} 3 \mathrm{H}, \mathrm{PeF}^{\prime} \mathrm{H}$, and PeANS in non-spot tissue. These results finally led to the lack of synthesis and accumulation of anthocyanin in non-spot area.

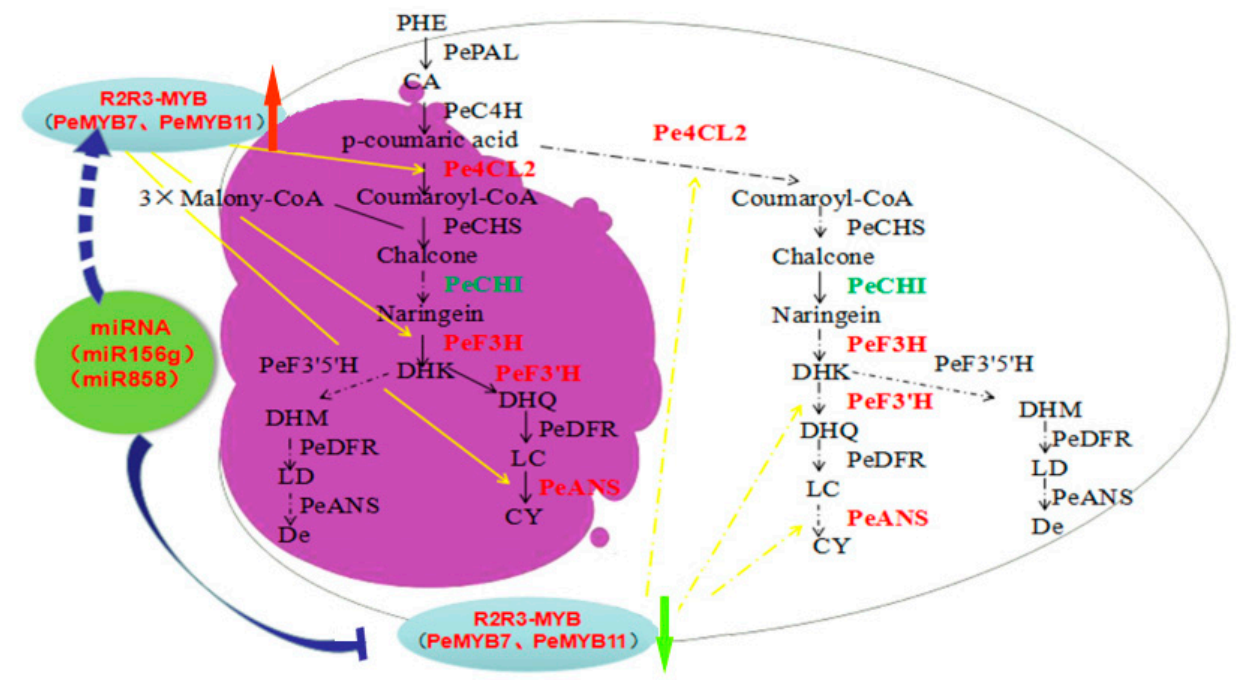

Figure 9. A proposed model summarizing of spot formation pattern in Phalaenopsis 'Panda' sepal. The purple segment illustrates the accumulation of anthocyanidin in spot area of 'Panda'. MIR156g, MIR858 target on key regulate genes PeMYB7 and PeMYB11, suppressed the transcript level of Pe4CL2, PeF3H, PeF3'H, PeANS and resulted in reduced anthocyanidin production in the non-spot area, while relatively lower expression levels of MIR156g and MIR858 and high levels of transcription of these genes in spot area cause accumulation of anthocyanidin. The black dashed arrows represent low levels of transcription, while the black solid-line arrows represent high levels of transcription. The yellow solid-line arrows represent promoting transcription, while yellow dashed arrow represent lack of promotion function. The blue bold solid-line T-arrow represents interfering transcription, while blue bold dashed arrow represents lack of interference function. The red bold arrow represents upregulation, and the green bold arrow represents downregulation. DHK, dihydrokaempferol; DHQ, dihydroquercetin; DHM, dihydromyricetin; LC, leucocyanidin; LD, leucodelphinidin; Cy, cyanidin; De, delphinidin.

\section{Materials and Methods}

\subsection{Plant Materials}

Phalaenopsis 'Panda' (Figure 10) were grown at the horticultural farm of Hainan University (Latitude: 20.03N, longitude: 110.33E), Haikou, Hainan Province, China. The sepal tissues were divided into two parts, spots area and non-spots area for total anthocyanin analyses and totally RNA extraction. Three independent biological replicates were collected for each sample. All samples were immediately frozen in liquid nitrogen and stored at $-80^{\circ} \mathrm{C}$ until use. 


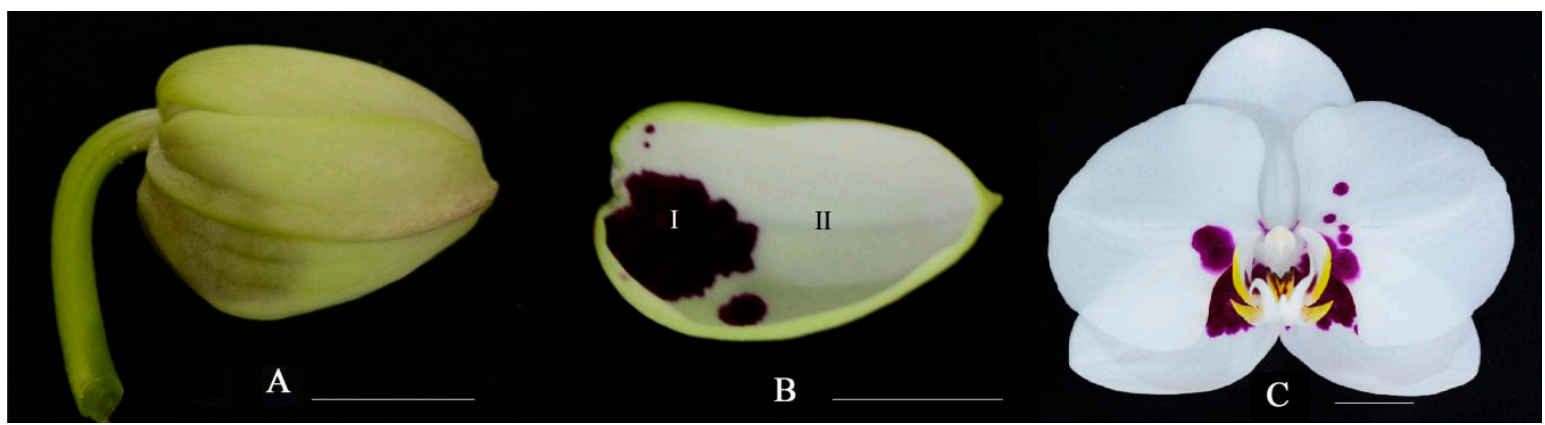

Figure 10. The flower tissue of Phalaenopsis 'Panda'. Bars $=1 \mathrm{~cm}$. (A) Full bud; (B) Sepal; I. spot area; II. non-spot area. (C) Full bloom stage.

\subsection{Observations of Sepal Anatomy and Determination of Total Anthocyanin Content}

Spot and non-spot sepal tissue of full bud were cut into segments longitudinally (approx.10 $\mathrm{mm} \times 5 \mathrm{~mm}$ ), and the upper epidermis and dorsal epidermis were separated with forceps. The sections were observed and photographed with a light microscope (Primo Vert, Zeiss, Germany).

The total anthocyanin content of Phalaenopsis sepal in spot and non-spot areas were determined by the $\mathrm{pH}$ differential method [36].

\subsection{RNA Extraction, cDNA Library Construction, and mRNA Sequencing}

RNAs were extracted from spot and non-spot parts of sepals of full bud using the modified Trizol method [6]. Two biological replicates were used for spot sepal and non-spot sepal. A total of 4 RNA-seq libraries were constructed from these sepals. RNA-Seq libraries were constructed using the RNA Library Prep Kit for Illumina according to the manufacturer's instructions (NEB, Ipswich, MA, USA). Library quality was assessed on the Agilent Bioanalyzer 2100 system. The mRNA libraries were sequenced on the Illumina HiSeq 2000 platform (Illumina, San Diego, CA, USA) based on sequencing by synthesis with 150 bp paired-end reads (Biomarker Technologies, Wuhan, China).

\section{4. mRNA Transcriptome Data Analysis}

The reads with adaptors were removed firstly. The reads in which unknown bases comprised more than $5 \%$ of the total and low quality reads (the percentage of the low quality bases of quality value $\leq 15$ is more than $20 \%$ in a read) were also removed. The clean reads were aligned to Phalaenopsis equestris genome accessed from (https://www.ncbi.nlm.nih.gov/bioproject/192198) by HISAT2 (http://www.ccb.jhu.edu/software/hisat/index.shtml) [37].

Gene alignment rate was counted with Bowtie2 v2.2.5 (http://bowtie-bio.sourceforge.net/bowtie2/ index.shtml), gene and transcript expression levels were calculated with RSEM v1.2.12 (http://deweylab. biostat.wisc.edu/RSEM). The differentially expressed genes (DEGs) between spot and non-spot tissues were identified and filtered with the R package DESeq2 based on $\mid \log 2$ (foldchange) $\mid \geq 1$ and Padj $<0.05$ [38]. The heatmap displays of the Trimmed Mean of M-values (TMM) normalized against the Fragment Per Kilobase of transcripts per Million (FPKM) mapped reads were performed using the R package pheatmap (https:/cran.r-project.org/src/contrib/Archive/pheatmap/). The DGEs were imported into Blast2GO software v2.5 [39] and in-house perl scripts for gene ontology (GO) term analysis, while the KEGG pathways were assigned to the assembled genes using the online KEGG Automatic Annotation Server (KAAS, http://www.genome.jp/kegg/), and then the phyper function in $\mathrm{R}$ software was used for enrichment analysis with $F D R \leq 0.01$. To identify transcription factors, the assembled transcriptome were searched against the Plant Transcription Factor Database PlnTFDB (http://plntfdb.bio.uni-potsdam.de/v3.0/downloads.php) using hmmseach v3.0 (http://hmmer.org). 


\section{5. $q R T-P C R$ Analyses of $m R N A$}

10 DEGs in anthocyanin biosynthesis and 4 DEGs of transcription factor were chosen for qRT-PCR analyses. qRT-PCR analyses were performed using SYBR Premix Ex TaqTM II (Tli RNaseH Plus) (Takara, Dalian, China) according to the manufacturer's instructions with the following reaction conditions: denaturation at $95^{\circ} \mathrm{C}$ for $30 \mathrm{~s}$ and 40 cycles of amplification $\left(95^{\circ} \mathrm{C}\right.$ for $5 \mathrm{~s}, 60^{\circ} \mathrm{C}$ for $\left.30 \mathrm{~s}\right)$. The $\beta$-Actin gene was used as an internal control for normalization. Relative expression levels of target genes were calculated using the $2^{-\Delta \Delta C t}[40]$ against the internal control. The gene-specific primers are shown in Table S7. Experiments were performed with three independent biological replicates and three technical replicates.

\subsection{Small RNA Library Construction and Sequencing}

RNA was extracted from spot and non-spot sepal using the modified Trizol method [6], and 4 small RNA libraries from sepal spot and non-spot samples with 2 biological replicates were constructed and sequenced by SBQ500 sequencing method (BGI, Wuhan, China). Then the low-quality, $5^{\prime}$ primer contaminants, without $3^{\prime}$ primers and insert tags, and sequences fewer than 18 nucleotides (nt) were filtered out to obtain clean reads from raw data reads. The final clean reads of the 4 libraries were mapped to Phalaenopsis genome and other smallRNA databases using Bowtie2 [41]. All the remaining clean sequences were annotated into different classes to remove rRNA, scRNA, snoRNA, snRNA, and tRNA using miRBase, siRNA, piRNA, snoRNA database with Bowtie2 [42] and Rfam database with cmsearch [43]. The novel miRNAs were predicted by Mireap software (https://sourceforge.net/ projects/mireap/).

\subsection{Data Analysis of Small RNA Sequencing}

The expression level of miRNAs was compared between spot and non-spot tissues to identify differentially expressed miRNAs (DEMs). The differentially expressed miRNA between spot and non-spot tissues were identified and filtered with the R package DESeq2 and Poisson Distribution [38,44]. The DEMs were determined based on FDR $\leq 0.001$ and the absolute value of $|\log 2 \mathrm{FC}| \geq 1$. The heatmap displays of the Trimmed Mean of M-values (TMM) normalized against the Fragment Per Kilobase of transcripts per Million mapped reads (FPKM) were performed created using the R package pheatmap (https://cran.r-project.org/src/contrib/Archive/pheatmap/).

The potential target genes by differentially expressed miRNAs were predicted and analyzed with 2 different software, including psRobot [45] and Targetfinder [46]. In order to increase the level of confidence and get more reliable results, we selected only those binding sites that were predicted by both of two softwares.

All protein-coding target genes regulated by DEMs were annotated against the KEGG databases. The KEGG enrichment analysis for the target genes of the DEMs was performed by Fisher's exact test (P-values) in Blast2GO pipeline, and P-values were used to conduct multiple test correction by FDR. KEGG terms with FDR $<0.05$ were considered to be significantly enriched.

\subsection{Verification of miRNA Expression Levels by $q P C R$}

Two miRNA targets on key regulate gene transcription factor PeMYB11 and PeMYB7 were chosen for qRT-PCR analyses. The qRT-PCR analyses were performed using SYBR Premix Ex TaqTM II (Tli RNaseH Plus) (Takara, Dalian, China) according to the manufacturer's instructions with the following reaction conditions: Denaturation at $95^{\circ} \mathrm{C}$ for $30 \mathrm{~s}$ and 40 cycles of amplification $\left(95^{\circ} \mathrm{C}\right.$ for $5 \mathrm{~s}, 60^{\circ} \mathrm{C}$ for $30 \mathrm{~s}$ ). The U6 gene was used as an internal control for normalization. Relative expression levels of target genes were calculated using the $2^{-\Delta \Delta \mathrm{Ct}}$ [40] against the internal control. The specific stem-ring primers were designed according to the miRNA qPCR primer design method [47], showed in Table S8. Experiments were performed with three independent biological replicates and three technical replicates. 
Supplementary Materials: Supplementary Materials can be found at http://www.mdpi.com/1422-0067/20/17/ 4250/s1.

Author Contributions: Conceptualization, J.W. and X.S.; methodology, A.Z., Z.C. and T.L.; software, H.P.; materies culture, Y.S. and X.L.; validation, A.Z., Y.Z. (Ying Zhao) and Y.Z. (Yang Zhou); writing一original draft preparation, A.Z.; writing-review and editing, J.W., W.H. and T.P.

Funding: This study was supported by the Hainan major science program (zdkj201815) and the National Natural Science Foundation of China $(31760590 ; 31260488)$. This research was conducted at Research Center for Terrestrial Biodiversity of the South China Sea, Hainan University, Hainan, China.

Conflicts of Interest: The authors declare no conflict of interest.

\section{References}

1. Eckhart, V.M.; Rushing, N.S.; Hart, G.M.; Hansen, J.D. Frequency-dependent pollinator foraging in polymorphic Clarkia xantiana ssp. xantiana populations: Implications for flower colour evolution and pollinator interactions. Oikos 2010, 112, 412-421. [CrossRef]

2. Miller, R.; Owens, S.J.; Rørslett, B. Plants and colour: Flowers and pollination. Opt. Laser Technol. 2011, 43, 282-294. [CrossRef]

3. Moeller, D.A. Pollinator community structure and sources of spatial variation in plant-pollinator interactions in Clarkia xantiana ssp. xantiana. Oecologia 2005, 142, 28-37. [CrossRef] [PubMed]

4. Shang, Y.; Venail, J.; Mackay, S.; Bailey, P.C.; Schwinn, K.E.; Jameson, P.E.; Martin, C.R.; Davies, K.M. The molecular basis for venation patterning of pigmentation and its effect on pollinator attraction in flowers of Antirrhinum. New Phytol. 2011, 189, 602-615. [CrossRef] [PubMed]

5. Chiou, C.Y.; Wu, K.; Yeh, K.W. Characterization and promoter activity of chromoplast specific carotenoid associated gene (CHRC) from Oncidium Gower Ramsey. Biotechnol. Lett. 2008, 30, 1861-1866. [CrossRef] [PubMed]

6. Li, Q.; Wang, J.; Sun, H.Y.; Shang, X. Flower color patterning in pansy (Viola x wittrockiana Gams.) is caused by the differential expression of three genes from the anthocyanin pathway in acyanic and cyanic flower areas. Plant Physiol. Biochem. 2014, 84, 134-141. [CrossRef] [PubMed]

7. Forkmann, G.; Dangelmayr, B. Genetic control of chalcone isomerase activity in flowers of Dianthus caryophyllus. Biochem. Genet. 1979, 18, 519-527. [CrossRef]

8. Matin, C.; Prescott, A.; Mackay, S.; Bartlett, J.; Vrijlandt, E. Control of anthocyanin biosynthesis in flowers of Antirrhinum majus. Plant J. 1991, 1, 37-49.

9. Holton, T.A.; Brugliera, F.; Tanaka, Y. Cloning and expression of flavonal synthase from Petunia hybrida. Plant J. 1993, 4, 1003-1010. [CrossRef]

10. Kawabata, S.K.Y.; Kusuhara, Y.K.; Li, Y.H.; Sakiyama, R.Z. The regulation of anthocyanin Biosythesis in Eustoma grandiflorum under low light condition. J. Jpn. Soc. Hort. Sci. 1999, 68, 519-526. (In Japanese) [CrossRef]

11. Bashandy, H.; Pietiainen, M.; Carvalho, E.; Lim, K.J.; Elomaa, P.; Martens, S.; Teeri, T.H. Anthocyanin biosynthesis in gerbera cultivar 'Estelle' and its acyanic sport 'Ivory'. Planta 2015, 242, 601-611. [CrossRef] [PubMed]

12. Ma, H.M.; Pooler, M.; Griesbach, R. Anthocyanin Regulatory/Structural Gene Expression in Phalaenopsis. J. Am. Soc. Hortic. Sci. 2009, 134, 88-96. [CrossRef]

13. Katsumoto, Y.; Fukuchi-Mizutani, M.; Fukui, Y.; Brugliera, F.; Holton, T.A.; Karan, M.; Nakamura, N.; Yonekura-Sakakibara, K.; Togami, J.; Pigeaire, A. Engineering of the rose flavonoid biosynthetic pathway successfully generated blue-hued flowers accumulating delphinidin. Plant Cell Physiol. 2007, 48, 1589. [CrossRef] [PubMed]

14. Saito, K.; Yonekura-Sakakibara, K.; Nakabayashi, R.; Higashi, Y.; Yamazaki, M.; Tohge, T.; Fernie, A.R. The flavonoid biosynthetic pathway in Arabidopsis: Structural and genetic diversity. Plant Physiol. Biochem. 2013, 72, 21-34. [CrossRef] [PubMed]

15. Zhao, L.; Gao, L.; Wang, H.; Chen, X.; Wang, Y.; Yang, H.; Wei, C.; Wan, X.; Xia, T. The R2R3-MYB, bHLH, WD40, and related transcription factors in flavonoid biosynthesis. Funct. Integr. Genom. 2013, 13, 75-98. [CrossRef] [PubMed] 
16. Chiou, C.Y.; Yeh, K.W. Differential expression of MYB gene (OgMYB1) determines color patterning in floral tissue of Oncidium Gower Ramsey. Plant Mol. Biol. 2008, 66, 379-388. [CrossRef] [PubMed]

17. Yamagishi, M. Oriental hybrid lily Sorbonne homologue of LhMYB12 regulates anthocyanin biosyntheses in flower tepals and tepal spots. Mol. Breed. 2011, 28, 381-389. [CrossRef]

18. Whang, S.S.; Wan, S.U.; Song, I.J.; Lim, P.O.; Choi, K.; Park, K.W.; Kang, K.W.; Mi, S.C.; Koo, J.C. Molecular Analysis of Anthocyanin Biosynthetic Genes and Control of Flower Coloration by Flavonoid 3', $5^{\prime}$-Hydroxylase $\left(\mathrm{F}^{\prime} 5^{\prime} \mathrm{H}\right)$ in Dendrobium moniliforme. J. Plant Biol. 2011, 54, 209-218. [CrossRef]

19. Martins, T.R.; Berg, J.J.; Blinka, S.; Rausher, M.D.; Baum, D.A. Precise spatio-temporal regulation of the anthocyanin biosynthetic pathway leads to petal spot formation in Clarkia gracilis (Onagraceae). New Phytol. 2013, 197, 958-969. [CrossRef]

20. Yamagishi, M.; Shimoyamada, Y.; Nakatsuka, T.; Masuda, K. Two R2R3-MYB genes, homologs of petunia AN2, regulate anthocyanin biosyntheses in flower tepals, tepal spots and leaves of Asiatic hybrid lily. Plant Cell Physiol. 2010, 51, 463-474. [CrossRef]

21. Hsu, C.C.; Chen, Y.Y.; Tsai, W.C.; Chen, W.H.; Chen, H.H. Three R2R3-MYB transcription factors regulate distinct floral pigmentation patterning in Phalaenopsis spp. Plant Physiol. 2015, 168, 175-191. [CrossRef]

22. Kaucsár, T.; Rácz, Z.; Hamar, P. Post-transcriptional gene-expression regulation by micro RNA (miRNA) network in renal disease. Adv. Drug Deliv. Rev. 2010, 62, 1390-1401. [CrossRef]

23. Koseki, M.; Goto, K.; Masuta, C.; Kanazawa, A. The star-type color pattern in Petunia hybrida 'red Star' flowers is induced by sequence-specific degradation of chalcone synthase RNA. Plant Cell Physiol. 2005, 46, 1879-1883. [CrossRef]

24. Guan, X.; Pang, M.; Nah, G.; Shi, X.; Ye, W.; Stelly, D.M.; Chen, Z.J. miR828 and miR858 regulate homoeologous MYB2 gene functions in Arabidopsis trichome and cotton fibre development. Nat. Commun. 2014, 5, 3050. [CrossRef]

25. Cai, J.; Liu, X.; Vanneste, K.; Proost, S.; Tsai, W.C.; Liu, K.W.; Chen, L.J. The genome sequence of the orchid Phalaenopsis equestris. Nat. Genet. 2015, 47, 65-72. [CrossRef]

26. Xu, L.; Yang, P.; Feng, Y.; Xu, H.; Cao, Y.; Tang, Y.; Yuan, S.; Liu, X.; Ming, J. Spatiotemporal Transcriptome Analysis Provides Insights into Bicolor Tepal Development in Lilium “Tiny Padhye". Front. Plant Sci. 2017, 8, 398. [CrossRef]

27. Li, C.Q.; Shen, W.; Yan, P.; Li, X.Y.; Zhou, P. Bioinformatic Prediction of microRNA and Their Target Gene in Mimulus gutatus. Guizhou Agric. Sci. 2015, 1, 8-12.

28. Zhang, Y.; Cheng, Y.; Ya, H.; Xu, S.; Han, J. Transcriptome sequencing of purple petal spot region in tree peony reveals differentially expressed anthocyanin structural genes. Front. Plant Sci. 2015, 6, 964. [CrossRef]

29. Hsu, C.C.; Su, C.J.; Jeng, M.F.; Chen, W.H.; Chen, H.H. A HORT1 retrotransposon insertion in the PeMYB11 promoter causes harlequin/black flowers in Phalaenopsis orchids. Plant Physiol. 2019, 180, 1535-1548. [CrossRef]

30. Hsu, C.C.; Chen, H.H. Flower Color and Pigmentation Patterns in Phalaenopsis Orchids. Orchid Biotechnology III; World Scientific Publishing: Singapore, 2017; pp. 393-420.

31. Bonar, N.; Liney, M.; Zhang, R.; Austin, C.; Dessoly, J.; Davidson, D.; Stephens, J.; McDougall, G.; Taylor, M.; Bryan, G.J.; et al. Potato miR828 Is Associated With Purple Tuber Skin and Flesh Color. Front. Plant Sci. 2018, 9, 1742. [CrossRef]

32. Gou, J.Y.; Felippes, F.F.; Liu, C.J.; Weigel, D.; Wang, J.W. Negative regulation of anthocyanin biosynthesis in Arabidopsis by a miR156-targeted SPL transcription factor. Plant Cell 2011, 23, 1512-1522. [CrossRef]

33. Chitwood, D.H.; Klein, L.L.; O'Hanlon, R.; Chacko, S.; Greg, M.; Kitchen, C.; Miller, A.; Londo, J.P. Latent developmental and evolutionary shapes embedded within the grapevine leaf. New Phytol. 2016, 210, 343-355. [CrossRef]

34. Jia, X.; Shen, J.; Liu, H.; Li, F.; Ding, N.; Gao, C.; Pattanaik, S.; Patra, B.; Li, R.; Yuan, L. Small tandem target mimic-mediated blockage of microRNA858 induces anthocyanin accumulation in tomato. Planta 2015, 242, 283-293. [CrossRef]

35. Ballester, A.R.; Molthoff, J.; Vos, R.D.; Hekkert, B.T.L.; Orzaez, D.; Fernándezmoreno, J.P.; Tripodi, P.; Grandillo, S.; Martin, C.; Heldens, J. Biochemical and molecular analysis of pink tomatoes: Deregulated expression of the gene encoding transcription factor SIMYB12 leads to pink tomato fruit color. Plant Physiol. 2010, 152, 71-84. [CrossRef] 
36. Giusti, M.M.; Wrolstad, R.E. Charaterization and Measurement of Anthocyanins by UV-Visible Spectroscopy. Food Anal. Chem. 2001. [CrossRef]

37. Kim, D.; Langmead, B.; Salzberg, S.L. HISAT: A fast spliced aligner with low memory requirements. Nat. Method 2015, 12, 357-360. [CrossRef]

38. Anders, S.; Huber, W. Differential expression analysis for sequence count data. Genome Biol. 2010, 11, R106. [CrossRef]

39. Conesa, A.; Götz, S. Blast2GO: A comprehensive suite for functional analysis in plant genomics. Int. J. Plant Genom. 2008, 2008, 619832. [CrossRef]

40. Livak, K.J.; Schmittgen, T.D. Analysis of Relative Gene Expression Data Using Real-Time Quantitative PCR and the $2^{-\Delta \Delta C t}$ Method. Methods 2001, 25, 402-408. [CrossRef]

41. Langmead, B.; Salzberg, S.L. Fast gapped-read alignment with Bowtie 2. Nat. Methods 2012, 9, 357-359. [CrossRef]

42. Langmead, B.; Trapnell, C.; Pop, M.; Salzberg, S.L. Ultrafast and memory-efficient alignment of short DNA sequences to the human genome. Genome Biol. 2009, 10, R25. [CrossRef]

43. Nawrocki, E.P.; Eddy, S.R. Infernal 1.1: 100-fold faster RNA homology searches. Bioinformatics 2013, 29, 2933-2935. [CrossRef]

44. Audic, S.; Claverie, J.M. The significance of digital gene expression profiles. Genome Res. 1997, 7, $986-995$. [CrossRef]

45. Wu, H.J.; Ma, Y.K.; Chen, T.; Wang, M.; Wang, X.J. PsRobot: A web-based plant small RNA meta-analysis toolbox. Nucleic acids Res. 2012, 40, W22-W28. [CrossRef]

46. Fahlgren, N.; Carrington, J.C. miRNA Target Prediction in Plants. Methods Mol. Biol. 2010, 592, 51-57.

47. Varkonyi-Gasic, E.; Hellens, R.P. qRT-PCR of Small RNAs. Methods in Molecular Biology; Humana Press: Totowa, NJ, USA, 2010; pp. 109-122.

(C) 2019 by the authors. Licensee MDPI, Basel, Switzerland. This article is an open access article distributed under the terms and conditions of the Creative Commons Attribution (CC BY) license (http://creativecommons.org/licenses/by/4.0/). 